\title{
Modelado inelástico de la primera dovela de puentes de voladizos sucesivos usando la normativa colombiana de puentes
}

\author{
Inelastic modelling of the first segment of balanced cantilever \\ bridges using colombian bridge codes
}

\author{
Cristian Alejandro Banda Montoya ${ }^{+}$(D) Julián Andrés Londoño \\ Henao $^{+(D)}$ y Carlos Alberto Riveros Jerez** (D) \\ + L\&L Ingenieros Consultores, Calle 32E No.80A-90, Medellín, \\ Colombia \\ * Escuela Ambiental, Universidad de Antioquia, Ciudad \\ Universitaria, Medellín, Colombia
}

\begin{abstract}
Resumen. Este artículo presenta un análisis comparativo de respuesta inelástica en la primera dovela de puentes construidos por el método de voladizos sucesivos, diseñados con base en los códigos colombianos de diseño de puentes CCP-14 y CCDSP-95. En el contexto nacional, no es común que los diseños de puentes incluyan el cálculo de ductilidad obtenida mediante modelado no lineal, es decir se asume un grado de ductilidad más no se verifica. Este aspecto es importante por cuanto un valor alto de deformación trae consigo una mejora considerable en la confiabilidad y la seguridad de un puente. Los resultados numéricos indican que la ductilidad por curvatura de la sección es aproximadamente 3 sin importar el código de diseño, un valor ajustado además a lo especificado en normativas internacionales. La curvatura obtenida en fluencia tiende a un valor similar, pero se tiene mayor ductilidad para la evaluación realizada según la norma CCP-14.
\end{abstract}

Palabras Claves. Puente de voladizos sucesivos; viga postensada; ductilidad; método de elementos finitos.

\begin{abstract}
This article presents a comparative analysis of inelastic response in the first superstructure segment of segmental bridges designed following the recommendations provided by the colombian bridge design standards CCP-14 and CCDSP-95. In the national context it is not common for bridge designs to include the calculation of ductility obtained from non-linear modeling, that is, a degree of ductility is assumed but not verified. A high value of deformation brings with it a considerable improvement in the reliability and safety of a bridge. The numerical results indicate that the curvature ductility of the section is approximately 3 regardless of the design code, a value also adjusted to that specified in international regulations. The curvature obtained at the yield level tends to a similar value, but ductility is larger for the evaluation carried out according to the design standard CCP-14.
\end{abstract}

Keywords. Segmental bridge; post-tensioned beam; ductility; finite element method.

Como citar. C. Banda, J. Londoño y C. Riveros, "Modelado inelástico de la primera dovela de puentes de voladizos sucesivos usando la normativa colombiana de puentes", Jou. Cie. Ing, vol. 12, no 1, pp. 


\section{Introduction}

La Norma Colombiana de Diseño de Puentes CCP-14 es la norma vigente en Colombia para el diseño y construcción de puentes [1]. Si bien esta norma entró en vigencia en el 2014, la mayor cantidad de puentes que se encuentran actualmente en operación, fueron diseñados y construidos bajo lineamientos del Código Colombiano de Diseño Sísmico de Puentes CCDSP-95 [2]. Parámetros asociados a temas hidráulicos, hidrológicos y socavación a considerar en el diseño de puentes a nivel nacional se encuentran regulados en el Manual de Drenaje para Carreteras [3]. Desde el punto de vista sísmico, los procedimientos presentados en la norma CCP-14 [1] para el diseño de los diferentes elementos que conforman un puente, se fundamentan en la teoría de la confiabilidad, donde mediante el uso de diferentes factores de carga, se asume cierta ductilidad para los elementos del puente, pero no se realiza su cuantificación. Si bien la norma CCP-14 [1] menciona el uso de métodos basados en desplazamientos con el propósito de realizar una evaluación cuantitativa de ductilidad de los diferentes elementos de un puente, no los incorpora como obligatorios, sino que los presenta como una recomendación. Salgado-Gálvez et al. [4] en un estudio de evaluación probabilística de la amenaza sísmica en Colombia utilizado en la norma CCP-14 [1], recomiendan explorar enfoques basados en niveles de desempeño, los cuales implican el uso de modelos no lineales, o enfoques de determinación de aceleraciones para determinación de fuerzas sísmicas como el propuesto por [5].

Casos de colapso de puentes por procesos constructivos inadecuados se encuentran en la literatura como el caso del puente Botan, proyectado para ser el puente construido mediante voladizos sucesivos más largo de Turquía, durante su construcción presentó el colapso de parte de su superestructura. Ozcelik y Tutus [6] presentan un estudio de las causas del colapso del puente Botan, resaltando la importancia de calibrar modelos con cambios medidos en obra para predecir el proceso constructivo de las dovelas, la reducción del movimiento del carro de avance de 5 días a 3 días, sin adecuadamente realizar el ajuste de la curva de curado del concreto, fue el detonante del colapso de parte de la superestructura. Wilches et al. [7] presentan un estudio de la evolución del diseño sísmico de puentes en Chile, este estudio es complementado con el análisis del desempeño de puentes en Chile durante el sismo de Maule en 2010. Wilches et al. [7] resaltan que el desempeño sísmico de un puente depende en gran forma en el tipo de suelo sobre el cual se encuentra construido. Ahmed and Aziz [8] presentan un estudio de la concepción y desarrollo de puentes segmentales enfatizando en los métodos constructivos donde se resaltan la rapidez constructiva por medio de segmentos o dovelas fundidas en sitio o prefabricadas, construcción en zonas ambientalmente sensitivas o con restricciones de acceso, y su facilidad constructiva dado que la superestructura puede ser construida desde la subestructura. En el caso de puentes segmentales construidos mediante voladizos sucesivos, Ahmed and Aziz [8] resaltan ventajas constructivas cuando se presentan limitaciones de acceso de segmentos prefabricados y su uso en puentes con luces hasta $230 \mathrm{~m}$, donde el diseño de la superestructura bajo los efectos de carga muerta y viva requiere que la superestructura se comporte como una estructura monolítica manteniendo estados admisibles de compresión o tensión del concreto para el estado de sevicio.

El comportamiento de la superestructura bajo los efectos de cargas dinámicas es complejo y se fundamenta en el concepto de permitir que algunos elementos de la superestructura entren en fluencia y disipen energía [8]. Por lo anteriormente expuesto, resulta de gran importancia, que en el momento de diseñar los diferentes elementos estructurales, la ductilidad sea considerada como un factor importante debido a que garantiza una condición de falla segura.

Es importante el comportamiento dúctil, teniendo en cuenta la fragilidad inherente del concreto, además se puede inferir que la generación de altos esfuerzos debe verse compensada con 
una adecuada disipación de energía mediante una deformación admisible. Adicionalmente, para evitar una posible condición de falla no segura, se debe disponer una cantidad suficiente de acero no tensado con el fin de obtener un valor de ductilidad adecuado y de esta forma poder disipar energía mediante deformaciones. La Figura 1. ilustra el fenómeno de falla, que por cuestiones de seguridad, se busca que ocurra en los elementos críticos que componen un puente.

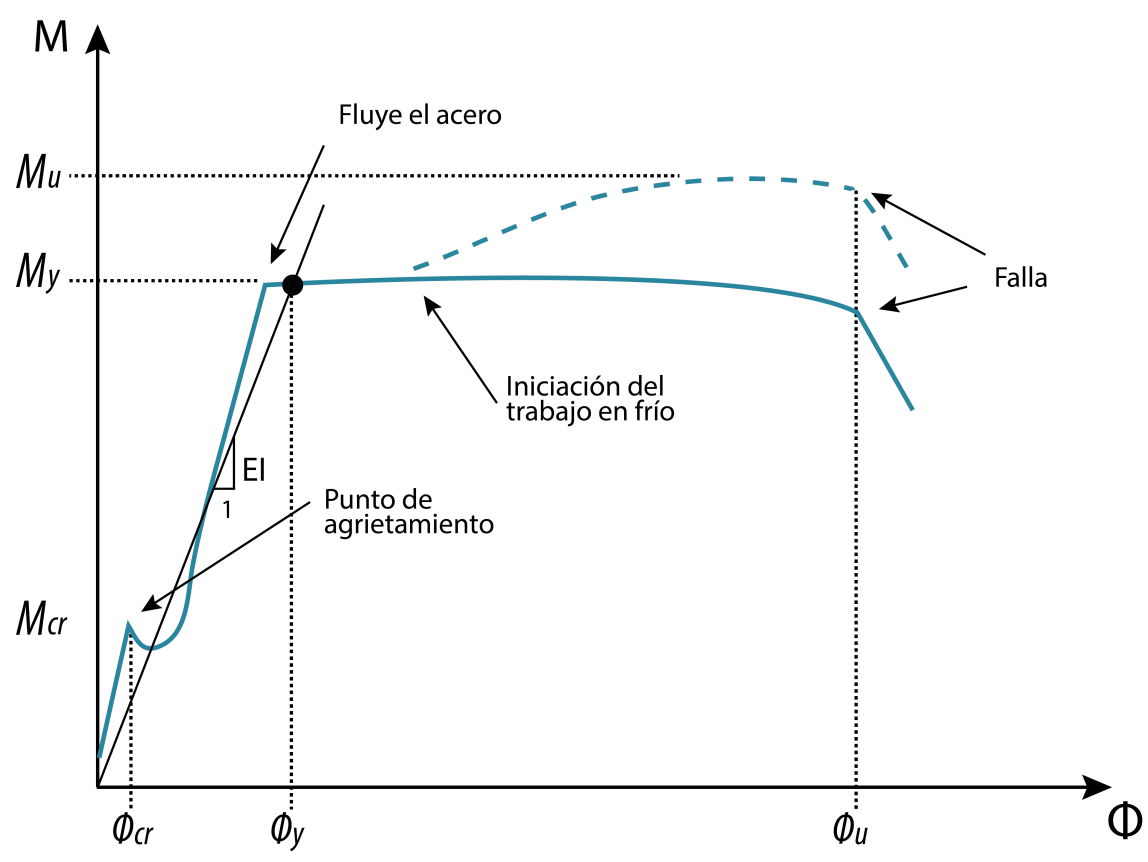

Figura 1: Puntos notables diagrama momento-curvatura. Fuente: Autores.

La Figura 1 muestra los puntos notables en un diagrama de momento-curvatura, el cual se compone de tres zonas, las cuales son: zona de respuesta elástica, descrita principalmente por el módulo de elasticidad del material, una zona sometida a tensión uniforme ubicada entre el punto de fluencia, el punto de endurecimiento del material y finalmente una zona de endurecimiento establecida entre el punto de endurecimiento del material y el punto de falla. Esta idealización del diagrama momento-curvatura, valora una fisuración nominal la cual no es de interés para el presente estudio, pues las solicitaciones que se deben atender superan el momento de fisuración.

El uso de diagramas de momento curvatura ha sido importante en el análisis de ductilidad y coeficientes de disipación de energía en puentes. Memari et al. [9] realizó un estudio de evaluación de ductilidad de columnas de puentes de concreto en la zona este de los Estados Unidos, el análisis de los diagramas de momento curvatura de las columnas de estos puentes arrojaron coeficientes de disipación de energía con valores entre 1.75 y 3. Una conclusión importante es que se debe realizar un estudio completo de respuesta del puente con el fin de determinar si fallas en columnas llevan al colapso del puente. Breccolotti et al. [10] estudiaron la ductilidad por curvatura de columnas cortas de concreto reforzado con carga biaxial, Breccolotti et al. [10] resaltan la dificultad que actualmente existe en la direccionalidad de la fuerza sísmica para la evaluación de los niveles de esfuerzo en columnas, resaltando que no existe un ángulo definido para determinar la ductilidad en columnas. Breccolotti et al. [10] resaltan también la importancia del recubrimiento del concreto en la determinación de ductilidad, ya que la pérdida del mismo lleva a una reducción de la ductilidad por curvatura, este aspecto es importante en el modelado de elementos de concreto reforzado para la determinación de ductilidad utilizando modelos como los propuestos en este artículo que modelan de forma independiente el concreto confinado y no 
confinado.

Farguier [11] presenta un estudio de colapso del viaducto 1 localizado en Venezuela, utilizando información geotécnica recolectada desde 1987 hasta la fecha de colapso del puente en 2006 y un estudio de todos los factores que influyeron en el colapso del puente, Farguier [11] recomienda el uso de diagramas de momento curvatura como una herramienta de gran importancia para investigar las rotaciones plásticas de secciones de concreto presforzado, también recomienda el uso de los valores mínimos y máximos de las rotaciones plásticas como herramienta potencial para la toma de decisiones debido al gran número de variables involucradas en el análisis, entre las cuales se tienen propiedades de los materiales, longitudes de rótulas plásticas, cargas, y valores de esfuerzo en el acero tensado. Wang y Zhai [12], en un estudio de evaluación de desempeño de puentes existentes de viga cajón, resaltaron la importancia de la ductilidad en la evaluación de fatiga en este tipo de puentes, Wang y Zhai [12] también resaltaron la importancia de realizar un estricto control del nivel de corrosión del acero de refuerzo como parámetro clave para controlar el desempeño de puentes por fatiga, este aspecto resalta la importancia de tener una línea base o parámetro inicial de referencia de ductilidad con el fin de evaluar posibles afectaciones causadas por fenómenos como la corrosión del acero de refuerzo, esta recomendación cobra especial importancia dado que entre los principales daños identificados en puentes existentes en Colombia se tiene la corrosión en vigas de concreto reforzado o presforzado [13].

Dhakal y Moustafa [14] recomiendan el uso de diagramas de momento curvatura para el diseño de vigas de concreto con materiales avanzados y de igual forma explorar nuevas alternativas de diseño para aplicaciones como puentes de vigas prefabricadas. Dolati and Maleki [15] realizaron un estudio de ductilidad para puentes de viga cajón de acero y enfatizan en la dificultad que se tiene para realizar reparaciones de puentes después de sismos de considerable magnitud por lo que recomiendan en uso de elementos de fácil reemplazo en la superestructura o entre la superestructura y la subestructura con el propósito de acomodar daños. Dolati and Maleki [15] plantearon un estudio de ductilidad mediante la determinación del coeficiente de disipación de energía (R), llegando a una propuesta de elemento que permite reducir la carga sísmica transmitida a la subestructura del orden de 3 a 4 . Con base en las anteriores investigaciones se resalta la importancia del uso de los diagramas de momento curvatura en el análisis de columnas y vigas de puentes, adicionalmente se debe considerar que estudios de falla local en estos elementos se deben complementar con estudios de falla global.

Desde el punto de vista nacional, es importante resaltar que los proyectos de construcción de infraestructura de 4ta Generación (4G) de concesiones viales han requerido de la construcción de puentes con especificaciones superiores a los existentes, por lo tanto se han requerido puentes con luces mayores en zonas de topografía escarpada, con el fin de cumplir con las especificaciones de diseño de estas vías, por este motivo, se han desarrollado estos nuevos puentes utilizando el método constructivo de voladizos sucesivos [16]. El tipo de proceso constructivo que se realice para la superestructura influye en gran manera sobre la subestructura, es por esto que el diseñador estructural debe concebir un puente que valore el tipo de andamiaje a utilizar o si se usará un carro de avance para el hormigonado de los voladizos sucesivos; la velocidad del viento en la zona de construcción, el gradiente de temperatura y calificación de la zona sísmica pues este último aspecto influye en la inclusión o no de aisladores sísmicos. Adicionalmente, se debe prever que los apoyos estén diseñados para el momento de vuelco que se pueda presentar debido a la construcción y los fenómenos que se puedan presentar antes de completar la unión de la ménsula en la luz central, la condición de continuidad generada por la unión de la luz central trae consigo una redistribución de momentos que debe estar concebida en los diseños de la viga en la zona cercana al apoyo pues el momento negativo disminuye para incrementar proporcionalmente el momento positivo en la luz central, debido a que se encuentra en un régimen estructural hiperestático.

Como ejemplos representativos de puentes de voladizos sucesivos a nivel nacional se tiene 
el puente Juanambú, que corresponde al primer puente construido bajo el sistema de voladizos sucesivos, llegando al punto de destacarse como premio nacional de ingeniería en el año 1972 y el segundo ejemplo corresponde al puente Roncador, el cuál para su terminación en el año 2020, será el puente de voladizos sucesivos más largo de Colombia. Debido a esta situación, es importante analizar los procedimientos recomendados por los códigos que han sido utilizados para el diseño y construcción de estos puentes. Adicionalmente, en áreas que se encuentran ubicadas en suelos susceptibles a licuefacción o cercanas a fallas geológicas, las cuales resultarían más afectadas ante la acción de sismos, la ductilidad constituye una consideración de extrema importancia, debido a que las normativas actuales en la mayoría de los países, plantean el diseño de estructuras que únicamente resistan los sismos moderados de manera elástica, y se confía en la disponibilidad de ductilidad de la sección después de su incursión en el rango inelástico para sobrevivir sin su colapso. Se tiene entonces como consecuencia que las recomendaciones se justifican sólo si la estructura tiene la suficiente ductilidad para absorber y disipar energía mediante deformaciones que no se presentan en el rango elástico [17].

\section{Análisis de Respuesta Inelástica}

Con el objetivo de aportar en mejoras que posiblemente se requieran para puentes existentes que fueron diseñados antes de la vigencia de la norma CCP-14 [1], este artículo presenta un procedimiento de análisis de respuesta inelástica de vigas cajón postensadas, estas vigas corresponden a dos puentes de voladizos sucesivos, los cuales fueron diseñados con base en recomendaciones de la norma CCP-14 [1] y el código CCDSP-95 [2], respectivamente. El primer puente se define como puente $\mathrm{A}$, diseñado con requerimientos sísmicos de zona de amenaza sísmica baja, de acuerdo a la tabla de categoría de comportamiento sísmico en el numeral A.3.5.3.2 del código CCDSP-95 [2], se determinó que el puente A se clasifica con un análisis CCS-C y un procedimiento mínimo de análisis sísmico PAS-2, según lo recomendado en el numeral A.3.5.4.3 [2].El puente A se encuentra en una región tipo 4, para la cual se tiene un valor de $\mathrm{Aa}=0.15$ [2]. Después de determinar el valor de Aa, se determinan los efectos de sitio, para esto se emplea la información suministrada en el estudio geotécnico en el cual se indica que el perfil de suelo corresponde a un perfil tipo S3, en consecuencia a esto y de acuerdo con la tabla A.3.5-1 del código CCDSP-95 [2] se determina que el coeficiente de sitio tiene un valor $\mathrm{S}=1.5$ [2], el cual corresponde a un perfil de suelo en donde entre la roca y la superficie hay más de $20 \mathrm{~m}$ de suelo que contiene depósitos estables de arcillas cuya dureza varía entre mediana y blanda, con una velocidad de la onda de cortante entre 150 y $270 \mathrm{~m} / \mathrm{s}$, y que dentro de ellos, en conjunto, hay menos de $12 \mathrm{~m}$ de arcillas blandas [2]. El puente A tiene una longitud total de 285 $\mathrm{m}$ y una sección de dovela inicial de $7 \mathrm{~m}$ de alto, se plantea que la viga cajón esté empotrada en los apoyos intermedios y que los estribos sean simplemente apoyados.

Los apoyos intermedios se encuentran apoyados sobre cimentaciones profundas que tienen una carga máxima por pilote de $5759 \mathrm{kN}$ y una carga admisible de $6000 \mathrm{kN}$, con el fin de garantizar estabilidad, se consideró una longitud de $35 \mathrm{~m}$ para los pilotes, los cuales se disponen por apoyo 8 pilotes en total, para los cuales se anticipa un desconfinamiento de $8 \mathrm{~m}$ aproximadamente debido a los procesos de socavación local del cuerpo de agua. El Nivel de Agua Máximo Esperado, N.A.M.E., asociado a un periodo de retorno de 100 años requiere de un gálibo de $5.25 \mathrm{~m}$ para el puente, cumpliendo de esta forma con lo establecido en el Manual de Drenaje para Carreteras [3]. Para los estribos, se proyectan 2 pilotes con $23 \mathrm{~m}$ de longitud cada uno, estos se deben proteger ante los efectos de socavación con un enrocado. En general los suelos que componen el cauce son de tipo cohesivos, compuestos por limos y arcillas de baja a media plasticidad.

El río tiene la capacidad de socavar la sección pero también de sedimentarla, en el caso de los estribos por estar posicionados en la llanura de inundación. Para evitar la socavación en los estribos, se propone limitarla mediante la protección del lecho y de las bancas del cauce contra las fuerzas erosivas del flujo de agua mediante protección local. La sección transversal del puente 
es una viga cajón postensada de altura variable con un tablero superior de $11.7 \mathrm{~m}$ de ancho. La Figura 2 presenta la geometría de la primera dovela del puente A.

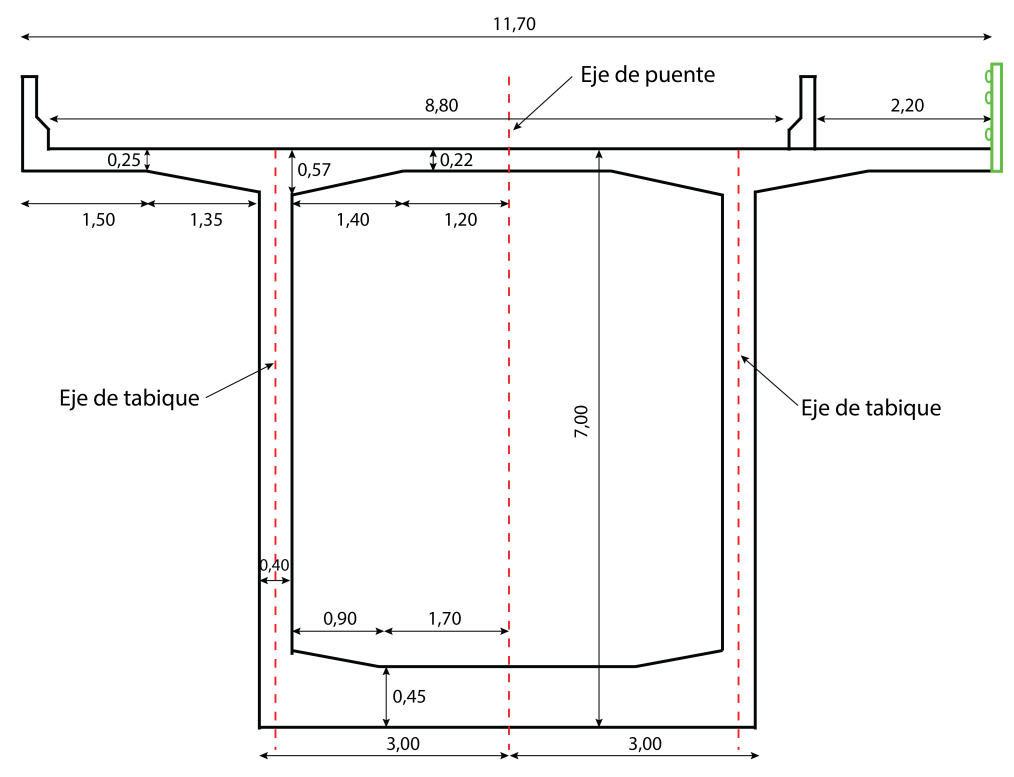

Figura 2: Sección transversal viga cajón puente A. Fuente: Autores.

El segundo puente se define como puente $B$, diseñado con requerimientos sísmicos correspondientes a una zona de amenaza sísmica intermedia, el sitio del puente se le puede asociar un perfil tipo C [1] y según la posición de los apoyos del puente frente a los afluentes de agua que se encuentran en la zona, se evidencia que estos se encuentran por fuera de la influencia de los cuerpos de agua, alejándolos de procesos de socavación. El perfil adoptado permite agrupar de forma coherente la tendencia general de los valores de la velocidad de onda. Iniciando en superficie, los primeros metros de profundidad se presenta un suelo residual que relaciona bajas velocidades de corte con un valor promedio de $350 \mathrm{~m} / \mathrm{s}$. A continuación, desde aproximadamente $2 \mathrm{~m}$ hasta $15 \mathrm{~m}$ se presenta en la estratigrafía un material de $400 \mathrm{~m} / \mathrm{s}$ relacionado con una capa de aglomerados y cenizas volcánicas, siguiendo hasta los $30 \mathrm{~m}$ donde aparece macizo de baja calidad correlacionado con un nivel de saprolito de $700 \mathrm{~m} / \mathrm{s}$ y finalmente roca fracturada con velocidades mayores a los $1000 \mathrm{~m} / \mathrm{s}$. Con base en el informe de amenaza sísmica se determinan los parámetros para la construcción del espectro de diseño según la norma CCP-14 [1]. Estos valores fueron determinados de acuerdo con la diversidad y complejidad del grupo de fallas geológicas que interfieren en la zona, desarrollando un estudio de respuesta sísmica local, con el objetivo de analizar la amenaza sísmica para el puente en específico. El puente B tiene una longitud total de $760 \mathrm{~m}$ y una sección de dovela inicial de $8.5 \mathrm{~m}$ de alto y $10.3 \mathrm{~m}$ de ancho; se plantean 5 apoyos intermedios con una luz entre ejes y longitud de columna variable, y estribos en los extremos del puente tipo pile bent, la longitud y cantidad de los pilotes varía entre $18 \mathrm{~m}$ a $34 \mathrm{~m}$ y 6 pilotes a 14 pilotes, respectivamente. La solicitación máxima por pilote es de $15461 \mathrm{kN}$ para una carga admisible de $15624 \mathrm{kN}$. Igualmente que en el puente A, la viga estará empotrada en los apoyos intermedios y se convertirá en un elemento continuo una vez se terminé el proceso de cosido mediante los cables de la losa inferior. La Figura 3 presenta la geometría de la primera dovela del puente B.

El procedimiento de respuesta inelástica presentado en este artículo tendrá como base la norma CCP-14 [1] y el código CCDSP-95 [2]. El problema se abordará modelando la primera dovela de cada puente en el software SeismoStruct [18], los parámetros de diseño sugeridos por 


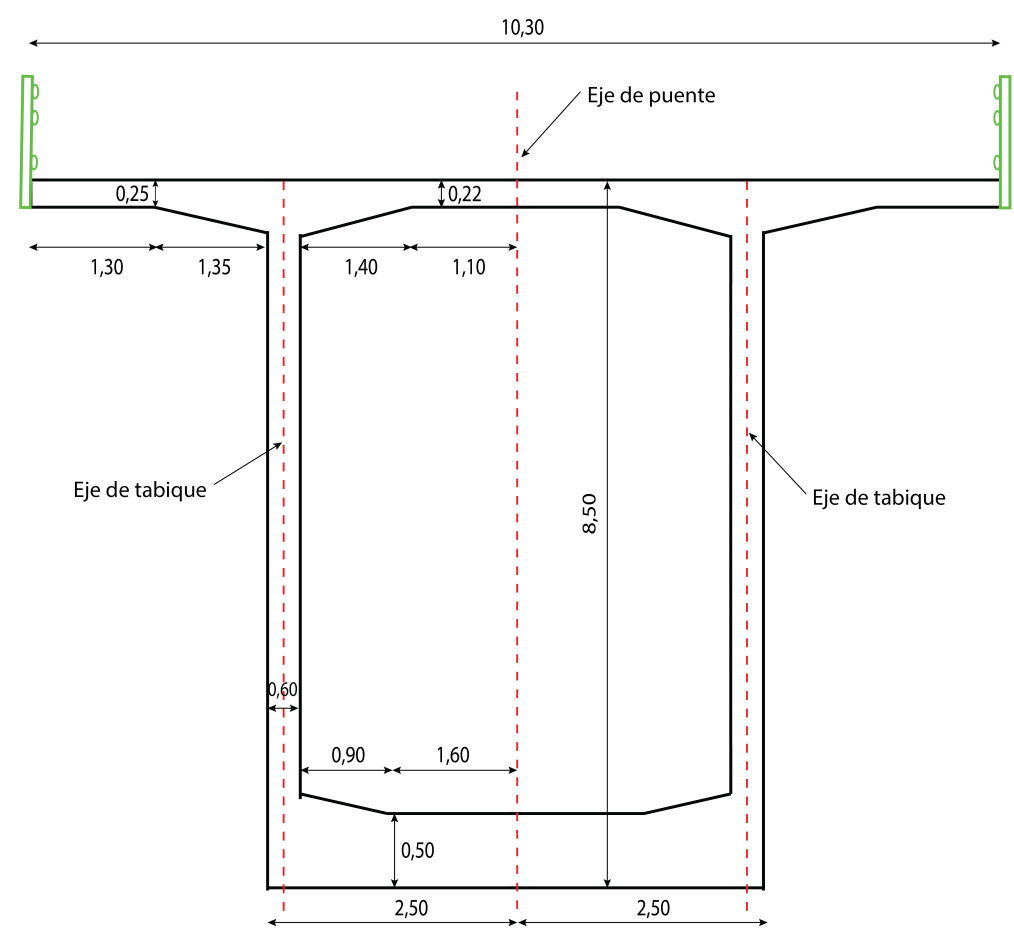

Figura 3: Sección transversal viga cajón puente B. Fuente: Autores.

cada metodología, una viga cajón postensada empleada en un puente con un obstáculo natural a vencer y posteriormente se realizará una comparación entre los resultados obtenidos por los diferentes enfoques de análisis estudiados.

\section{Parámetros de Modelado de Respuesta Inelástica}

Es indispensable contextualizarse sobre la importancia de garantizar adecuada ductilidad, en las conexiones entre los elementos horizontales y verticales que componen la estructura de un puente. El modelo constitutivo del concreto propuesto por Mander [19] considera la variación en resistencia que presenta el concreto al confinarse con elementos de acero de forma rectangular, mejorando de esta forma las características esfuerzo deformación, este modelo se considera adecuado para un análisis de momento-curvatura según Priestley [20]. El valor del momento de fisuración $M_{c r}$, es un valor que se puede calcular con base en el módulo de rotura $f_{r}$; pero es importante resaltar que el valor del módulo de rotura $f_{r}$ difiere dependiendo del código de diseño que se esté usando. Con el paso del tiempo, se ha logrado la implementación de distintos sistemas constructivos, que hoy en día proporcionan eficiencia al material a nivel estructural buscando la disminución del agrietamiento que presenta el concreto por su naturaleza, con el uso de diferentes procedimientos es posible determinar la contribución en términos de resistencia y ductilidad del refuerzo presforzado o postensado en vigas de concreto [21], [22]. En el caso de concreto postensado, con la aplicación de carga inducida exteriormente al concreto, los esfuerzos de tensión disminuyen y/o se anulan, y esto a su vez permite el mejoramiento estructural de un elemento; así el material se mantiene bajo tensiones admisibles.

Es necesario determinar los niveles de daño del elemento mediante rangos que reflejen la magnitud de estos daños y que den lugar a un desempeño predecible; este desempeño se puede cuantificar en términos de la cantidad de daño sufrido por afectaciones debidas a un evento extremo y el impacto que tienen estos daños en las actividades posteriores al evento extremo [23]. Estos niveles de daño, se pueden limitar con valores de deformaciones unitarias que presentaría 
el acero cuándo es sometido a esfuerzos. Con base en lo anteriormente expuesto, en el presente estudio se determinó un intervalo limitado por la deformación de fluencia del acero y por el valor asumido de deformación última del acero. Cuando el acero llega a una deformación tal que entra en fluencia, se encuentra ubicado en uno de los puntos notables del diagrama momento curvatura, diagrama que resulta de mucha utilidad para evaluar fisuramiento, además se puede hacer una evaluación de la ductilidad con base en la curvatura de una sección; analizando la fluencia y las condiciones ultimas de esta. La elaboración del diagrama momento-curvatura se realiza con la ayuda del software SeismoStruct [18], el cual además permite evaluar las condiciones últimas de la sección mediante el momento último que puede resistir.

\section{Modelado de Respuesta Inelástica}

A continuación, se presenta la verificación de ductilidad para las secciones de los dos puentes, el primer puente a evaluar es el puente A que se encuentra diseñado con base en el código CCDSP-95 [2]; el segundo puente a evaluar, que corresponde al puente B, se encuentra diseñado con base en la norma CCP-14 [1]. Se evaluará la ductilidad por curvatura de la sección de la primera dovela que compone cada uno de los puentes, el análisis se realiza sobre esta dovela debido a que es el elemento que se verá sometido a mayores solicitaciones y al ser el elemento conector con la columna corresponde al elemento más crítico de la superestructura. Para el logro de este objetivo, se ingresan al software parámetros como el módulo de elasticidad del concreto y el módulo de rotura, los cuales fueron estimados para cada código de diseño según la resistencia a compresión del concreto. El valor de resistencia a la compresión no debe variar del utilizado en el diseño $f^{\prime} c$, para este caso se tiene un valor de $42 \mathrm{MPa}$, empleando las Eq. (1) y Eq. (2) [2], se obtienen valores de $30740 \mathrm{MPa}$ y $4.06 \mathrm{MPa}$, para el módulo de elasticidad $E_{c}$ y módulo de rotura $f_{r}$, respectivamente. Además, se deben especificar las deformaciones esperadas en el punto de esfuerzo máximo según lo indicado por el código de diseño, en este caso se tiene un valor de 0.003 , y para el peso unitario del concreto convencional se tiene $24 \mathrm{kN} / \mathrm{m}^{3}$. Todos estos valores son ingresados para que el modelo constitutivo reflejado en el concreto tenga un comportamiento similar al especificado por el código CCDSP-95 [2].

$$
\begin{gathered}
E_{c}=15000 \sqrt{f^{\prime} c}\left[\frac{\mathrm{Kg}}{\mathrm{cm}^{2}}\right] \\
f_{r}=2 \sqrt{f^{\prime} c}\left[\frac{\mathrm{Kg}}{\mathrm{cm}^{2}}\right]
\end{gathered}
$$

Para el modelado numérico del acero de refuerzo se selecciona el modelo bilineal (stl_bl) que garantiza buena precisión en el modelado de la respuesta del acero de refuerzo y es uno de los más simples y eficientes modelos del acero de refuerzo [24]. El modelo bilineal (stl_bl), incorporado en el software Seismostruct [18], ha sido usado con éxito en estudios de respuesta de puentes [25]. Otros modelos constitutivos del acero de refuerzo han mostrado adecuado desempeño en determinar la respuesta del acero de refuerzo durante cargas cíclicas complejas [26] y han sido usados principalmente en estudios de determinación de demanda sísmica de puentes [27], [28]. Como el objetivo del presente estudio es obtener diagramas de momento-curvatura, el modelo bilineal (stl_bl), que no considera el efecto Bauschinger, se considera adecuado basado en recomendaciones de estudios donde se realizan análisis en puentes utilizando diagramas de momento-curvatura [29], [30].

Para este estudio se tiene un acero de refuerzo con valores de resistencia a la fluencia y módulo de elasticidad de $420 \mathrm{MPa}$ y $200 \mathrm{GPa}$, respectivamente. Se tienen deformaciones esperadas en el punto de endurecimiento y en el punto de falla con valores de 0.005 y 0.0125 , respectivamente. El modelo constitutivo del acero toma un valor de peso unitario de $78.5 \mathrm{kN} / \mathrm{m}^{3}$. La Figura 4 muestra la sección modelada para la primera dovela del puente A [18]. 


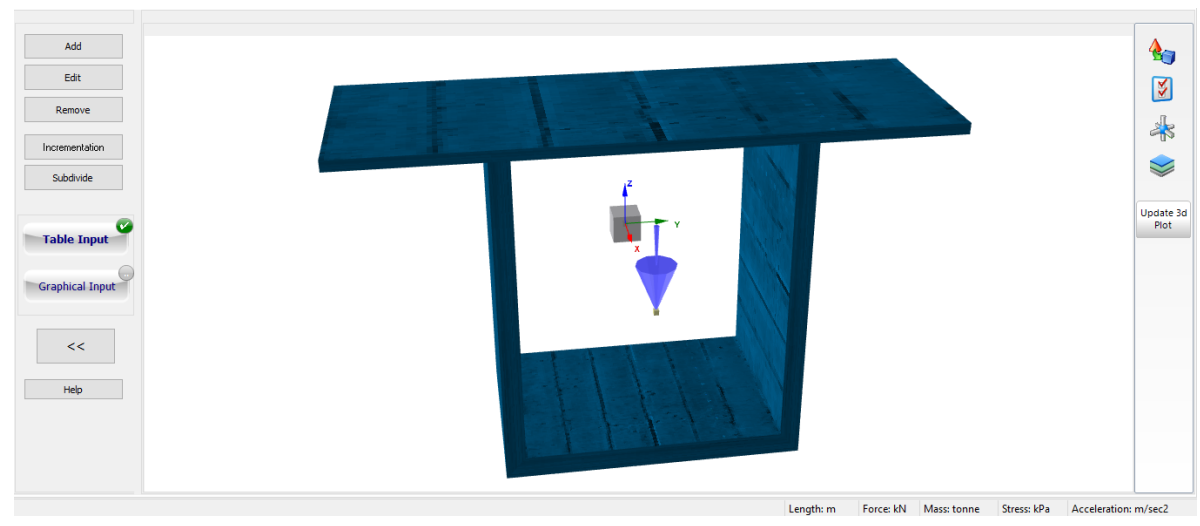

Figura 4: Modelo primer dovela puente A [18]. Fuente: Autores.

Para el acero postensado se tiene en cuenta una reducción en la resistencia producida por las pérdidas ocasionadas por la transferencia de las cargas externas, se hizo uso del valor de resistencia a la fluencia del acero postensado fpy $=1676 \mathrm{MPa}$, pues hasta este punto sigue guardando una cierta proporcionalidad entre el esfuerzo y la deformación. El área de acero postensado de la sección tiene efectos en las características del diagrama momento-curvatura y la ductilidad, cuando el acero postensado alcanza su deformación última (fractura), se tiene una reducción de momento con una alta curvatura [31].

Debido a la ubicación del elemento dentro de la superestructura, se restringen los grados de libertad en uno de los nodos simulando el empotramiento en el nudo. Para una correcta evaluación de los valores de deformación unitaria del acero y concreto; se define un intervalo al interior del software con el objeto de evaluar la respuesta del elemento en el momento de sufrir estas deformaciones. Se evalúa entonces la fluencia del acero con un valor de 0.0021, y para evaluar la falla un valor de 0.0075 , este valor se encuentra por encima de la deformación de endurecimiento. Se evalúa entonces la deformación aprovechable del concreto para verificar que se presente después de que el acero pase de condiciones últimas, y así comprobar que la condición de falla que se está presentando es la más adecuada y segura para el puente. Como se había enunciado anteriormente, se determinan el momento último que resiste la sección y el valor de ductilidad por curvatura. Para comenzar se toman los resultados numéricos obtenidos de la modelación numérica referentes al valor del momento alcanzado por la sección antes de la falla, estos resultados tienen la limitación de estar referenciados al factor de carga incremental que se ubica en el nodo para evaluar la flexión. Se tiene entonces un valor para el momento último de $918568 \mathrm{kN}-\mathrm{m}$ y un factor de carga de 225 según la Figura 5 . Se obtiene un valor de curvatura última de $0.001828141 / \mathrm{cm}$ y un valor de curvatura de fluencia de $0.00055181 \mathrm{1} / \mathrm{cm}$. Se realiza el análisis de ductilidad por curvatura de la sección para el análisis de comportamiento inelástico de la sección después de presentarse la fluencia, con los anteriores valores de referencia la ductilidad por curvatura de la sección, de la división entre la curvatura que tiene en el punto de ruptura y el punto de fluencia se obtiene un valor de 3.31298817.

Para la evaluación de la dovela con base en el CCP-14 [1], la sección no se modificó en cuanto a geometría y refuerzo no tensado, debido a que las consideraciones de los códigos varían respecto al acero tensado después de las pérdidas por la transferencia del preesfuerzo, la equivalencia entre la resistencia a la fluencia del acero y la resistencia efectiva después de pérdidas muestra como resultado un valor distinto al usado para el CCDSP-95 [2]. El modelo constitutivo del concreto permanece igual, por lo tanto, se deben calcular nuevamente valores como el módulo de elasticidad del concreto $E_{c}$ y el módulo de rotura $f_{r}$, pues en estos parámetros hay una discrepancia si se comparan las Eq. (1) y Eq. (2) [2]. Los valores de estos parámetros 


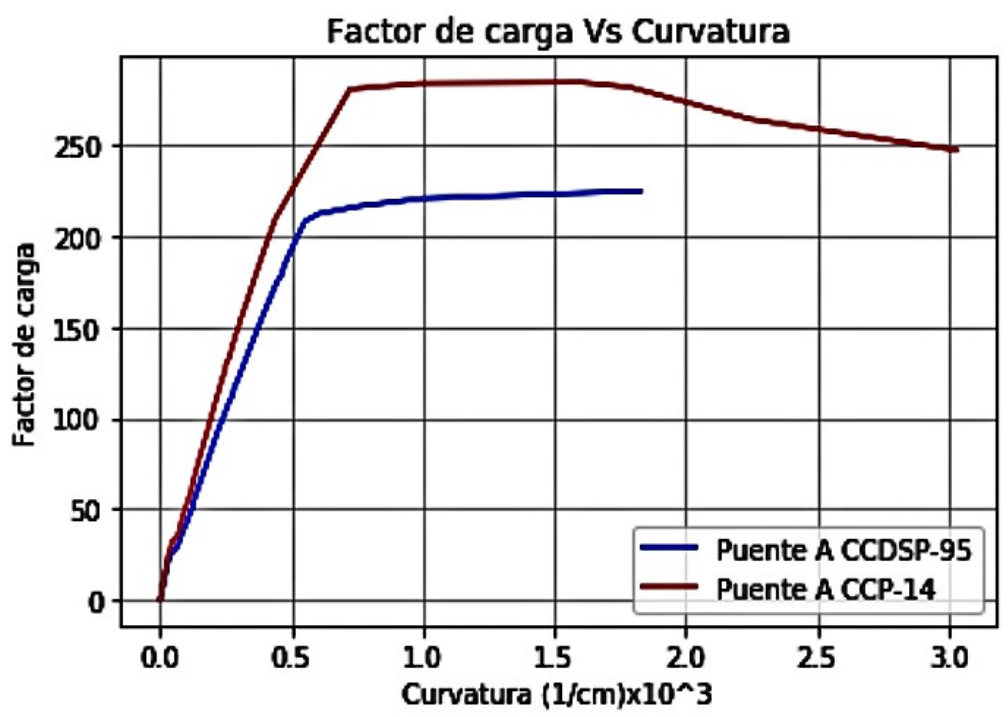

Figura 5: Factor de carga-curvatura puente A. Fuente: Autores.

se estimaron empleando las Eq.(3) y Eq. (4) [1], obteniendo valores de $30460 \mathrm{MPa}$ y 4.02 $\mathrm{MPa}$, respectivamente. El valor de resistencia a la compresión del concreto $f^{\prime} c$ no debe variar del utilizado anteriormente, se tiene entonces un valor de $f^{\prime} c=42 \mathrm{MPa}$, además, se deben especificar las deformaciones esperadas en el punto de esfuerzo máximo según lo indicado por el código de diseño, en este caso es un valor de 0.003 y el peso unitario del concreto convencional de $24 \mathrm{kN} / \mathrm{m}^{3}$. Todos estos valores son ingresados para que el modelo constitutivo reflejado en el concreto tenga un comportamiento similar al esperado por el CCP-14 [1].

$$
\begin{aligned}
E_{c} & =4800 \sqrt{f^{\prime} c}[\mathrm{MPa}] \\
f_{r} & =0.62 \sqrt{f^{\prime} c}[\mathrm{MPa}]
\end{aligned}
$$

El intervalo de deformaciones evaluado tiene como límite 0.0075 , debido a que en la norma CCP-14 sección 5.7.3.5 [1], específica claramente que, ante una redistribución de momentos, los momentos positivos deben ajustarse para tener en cuenta los cambios en los momentos negativos, con el fin de mantener el equilibrio de cargas y de fuerzas. Debido al proceso constructivo, la sección se ve obligada a tener que sufrir este fenómeno. Teniendo en cuenta la consideración para estos elementos, se plantea un rango de deformaciones unitarias en el acero para monitorear el mecanismo de falla que tiene el elemento. Se tiene entonces un límite inferior de 0.0021 para la fluencia, 0.0075 para la falla y con el objetivo de rectificar la condición de falla, se evalúa que el concreto entre en fluencia después que lo haya hecho el acero, para esto se toma un valor de 0.003 para la deformación unitaria en el concreto, de igual forma se analiza el momento último que resiste la sección según la norma CCP-14 [1]. Para comenzar se toman los resultados entregados por el modelo referentes al valor del momento alcanzado por la sección antes de la falla, estos resultados tienen como limitante que se encuentran referenciados al factor de carga incremental que se ubicó en el nodo para evaluar la flexión; se tiene entonces un valor para el momento último de $1026 \mathrm{MN}-\mathrm{m}$ y un factor de carga de 285 según la Figura 5. Si se realiza la comparación entre el valor obtenido anteriormente y este, se obtiene un porcentaje de diferencia del $10 \%$. Finalmente, se realizará el análisis de ductilidad por curvatura de la sección para el análisis de comportamiento inelástico de la sección después de presentarse la fluencia, para esto se tendrán dos puntos de referencia en el diagrama, los cuales son el punto de fluencia del acero 
con una deformación de 0.0021 y el punto de ruptura del acero con una deformación de 0.0075 . La ductilidad por curvatura de la sección se obtendrá de la división entre la curvatura que tiene en el punto de ruptura y el punto de fluencia. La curvatura última obtenida es $0.002248591 / \mathrm{cm}$ y la curvatura de fluencia es $0.000723231 / \mathrm{cm}$, para un valor de ductilidad por curvatura de 3.10909393. Se tiene entonces que la ductilidad por curvatura de la sección es aproximadamente 3 sin importar el código de diseño, un valor ajustado además a lo especificado en la normativa de la AASHTO LRFD Bridge Design Specifications [32]. Esto se debe a que, sin importar los parámetros de elasticidad de los elementos, la rigidez no afectará el punto de fluencia y la diferencia entre los valores obtenidos puede deberse a los ajustes de valores como ser el módulo de elasticidad y el módulo de rotura del concreto.

La rigidez proporcionada al elemento influye en los resultados, pues una vez la sección se ha visto flexionada, la resistencia tiene una leve dependencia de la rigidez, la suposición de una rigidez constante implica que la deformación de fluencia es directamente proporcional a la deformación causada por un momento actuante. Está suposición resulta invalida, debido a que esencialmente la rigidez es proporcional a la capacidad que tiene el elemento de deformarse, por tanto, la curvatura que alcanza durante la fluencia es independiente de su deformación. Por lo anteriormente expuesto se puede definir una analogía en que un valor alto de deformación trae consigo una mejora considerable en la confiabilidad y la seguridad de la estructura del puente. Por lo tanto, el hecho de haber inducido un valor de deformación mayor representa una capacidad última mayor ante solicitaciones de flexión. Considerando ambos normativas de diseño de puentes, se diferencian en el factor de carga generado para obtener la deformación de fluencia y la curvatura final alcanzada, el factor de carga obtenido no es indicativo de una gran diferencia en los códigos de diseño pues la rigidez proporcionada no hace una variación considerable a la curvatura de fluencia, por el contrario se puede observar claramente que la evaluación realizada según el CCP-14 [1], presenta una curvatura de falla mucho mayor a la obtenida por CCDSP-95 [2]. Teniendo presente, que la curvatura obtenida para la fluencia tiende a un valor similar se tiene entonces un rango entre la curvatura de fluencia y la curvatura de falla mucho mayor para la evaluación realizada según el CCP-14 [1].

Se procede a evaluar el segundo puente, que corresponde al puente $\mathrm{B}$, el cuál presenta una geometría diferente debido a que el vano que debe cubrir tiene una longitud mayor. La sección por evaluar presenta los mismos parámetros del puente A para el CCDSP-95 [2]; la longitud del elemento es menor y tiene una altura mayor. Para la evaluación se ingresaron nuevamente los valores al software SeismoStruct [18] según se ilustra en la Figura 6.

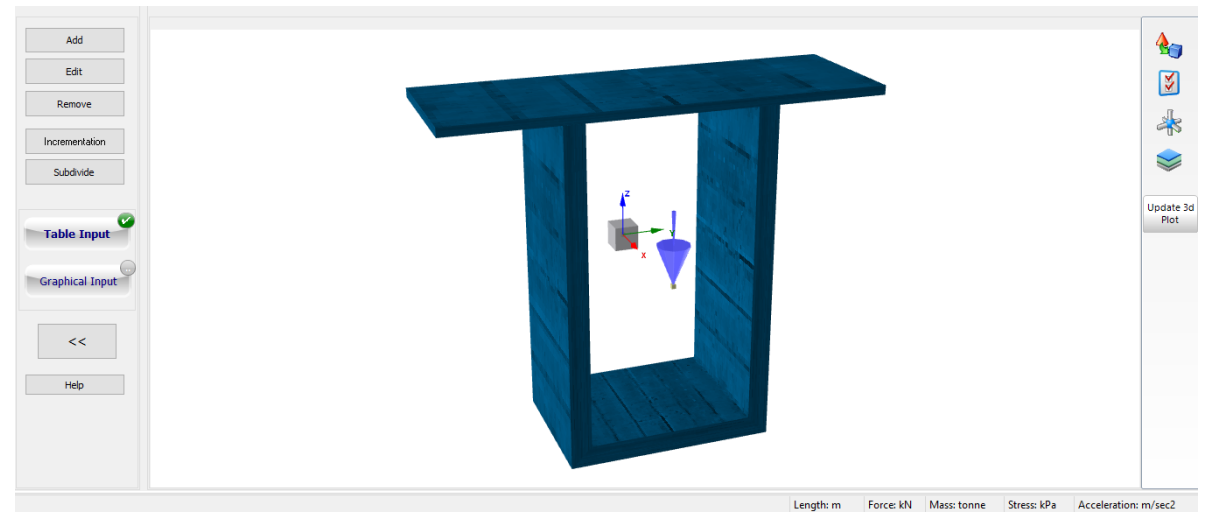

Figura 6: Modelo primer dovela puente B [18]. Fuente: Autores.

El valor de resistencia a la compresión no debe variar del utilizado para el diseño, se tiene entonces un valor de $f^{\prime} c=42 \mathrm{MPa}$, empleando las Eq. (1) y Eq. (2) [2], se obtienen valores 
de $30740 \mathrm{MPa}$ para el módulo de elasticidad $E_{c}$ y $4.06 \mathrm{MPa}$ para el módulo de rotura $f_{r}$. Además, se deben especificar las deformaciones esperadas en el punto de esfuerzo máximo según lo indicado por el código de diseño, en este caso es un valor de 0.003 y el peso unitario del concreto convencional de $24 \mathrm{kN} / \mathrm{m}^{3}$. Todos estos valores son ingresados para que el modelo constitutivo reflejado en el concreto tenga un comportamiento similar al esperado por el CCDSP-95 [2]. Se debe ingresar nuevamente el modelo constitutivo del acero, siguiendo el mismo tipo de material se realizó la modelación con el modelo constitutivo bilineal del acero de refuerzo (stl_bl) y se modificaron algunos valores para adaptar el modelo sugerido por el software al comportamiento esperado por el CCDSP-95 [2], se ingresaron los parámetros necesarios y que no difieran con lo empleado en los diseños estructurales, el módulo de elasticidad del acero tiene un valor de 200 GPa y la resistencia a la fluencia un valor de $420 \mathrm{MPa}$. Debido a que se deben ingresar las deformaciones esperadas en el punto de endurecimiento y en el punto de falla, no se modifican los valores ingresados anteriormente para el puente A, debido a que el software sugiere un valor de peso unitario para el acero de refuerzo, se toma entonces el valor de $78.5 \mathrm{kN} / \mathrm{m}^{3}$ y no se modifica. $\mathrm{El}$ valor de las deformaciones de estudio no difiere al usado anteriormente. Se toman los resultados entregados por el modelado numérico referentes al valor del momento alcanzado por la sección antes de la falla, estos resultados tienen como limitante que se encuentran referenciados al factor de carga incremental que se ubicó en el nodo para evaluar la flexión. Se tiene entonces un valor para el momento último de $976028 \mathrm{kN}-\mathrm{m}$ y un factor de carga de 218 como se ilustra en la Figura 7. A partir de la gráfica de factor de carga contra curvatura, se realiza el mismo análisis para hallar el valor de ductilidad por curvatura. Donde los valores obtenidos para la curvatura última y la curvatura de fluencia son $0.001322391 / \mathrm{cm}$ y $0.000438781 / \mathrm{cm}$, respectivamente. Finalmente se tiene un valor de ductilidad de 3.01378823 .

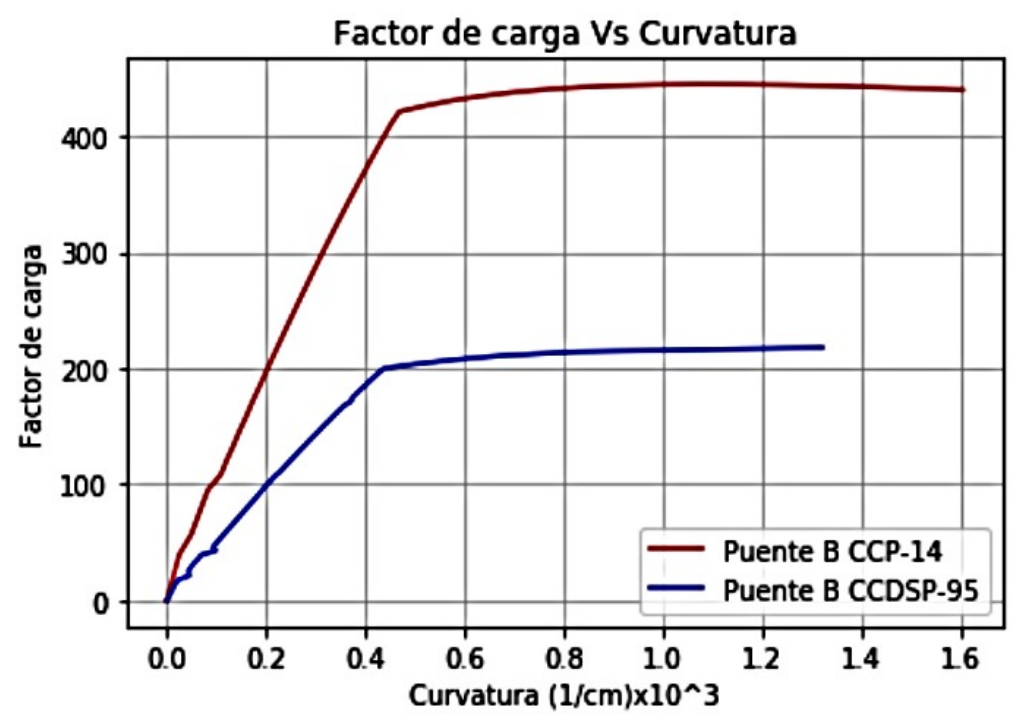

Figura 7: Factor de carga-curvatura puente B. Fuente: Autores.

Se realizará el mismo análisis para la norma código CCP-14 [1], con las consideraciones que este presenta ante este tipo de elementos; de igual manera que se hizo con el puente A. La geometría no difiere de la usada para evaluar el código CCDSP-95 [2] y se conservan los mismos parámetros usados anteriormente para que el modelo constitutivo reflejado en el concreto tenga un comportamiento similar al esperado por la norma CCP-14 [1]. Se toman los resultados obtenidos del modelo numérico referentes al valor del momento alcanzado por la sección antes de la falla, estos resultados tienen como limitante que se encuentran referenciados al factor de 
carga incremental que se ubicó en el nodo para evaluar la flexión; se tiene entonces un valor para el momento último de $1092 \mathrm{MN}-\mathrm{m}$ y un factor de carga de 445 según se ilustra en la Figura 9. De la misma forma con la que se ha evaluado la ductilidad en el puente A y en el CCDSP-95 [2], se evalúan los dos puntos notables del diagrama de momento-curvatura con el fin de establecer el estado límite de daño más conveniente para la seguridad y el óptimo funcionamiento de la estructura. Se tiene entonces los siguientes valores para la curvatura última y la curvatura de fluencia, $0.001431351 / \mathrm{cm}$ y $0.000461721 / \mathrm{cm}$ con los cuales se halla un valor para la ductilidad de 3.100038985. Se puede notar la afinidad de los resultados debido a los valores obtenidos en los diferentes códigos de diseño de puentes, además el valor obtenido para el momento último en el análisis del código actual resulta mayor que el obtenido en el CCDSP-95 [2], por las razones antes expuestas, se puede permitir el diseño bajo el CCDSP-95 [2] siempre y cuando este valore el cambio en el estado de esfuerzos debido a la redistribución de momentos. El valor de 0.0075 es un valor en el cual las fibras se encuentran en fluencia y la sección está plastificada, pero todavía hay una reserva de energía de la sección lo suficiente para indicar el estado de daño; está deformación es excesiva y no se tiene en cuenta en el análisis plástico pues no es necesario llegar a la rotura.

\section{Conclusiones}

Con los resultados presentados en este artículo, es posible afirmar que las secciones de los puentes estudiados tendrán un comportamiento adecuado ante la ocurrencia de un evento extremo, sin embargo, los diseños que se lleven a cabo en el país deberían velar porque los elementos estructurales que formen parte de los puentes presenten valores altos de deformación. Sin abandonar la esencia de diseño más actualizada, se pudo realizar la verificación de ductilidad para las secciones diseñadas; cumpliendo a cabalidad los requerimientos que dictan las dos normativas colombianas, demostrando así que las secciones presentadas son adecuadas para cualquier metodología que sea usada para el diseño de puentes a nivel mundial.

Respecto a la responsabilidad estructural conferida a la primera dovela, con base en los resultados obtenidos, es posible evidenciar un comportamiento en un rango de deformación suficiente para que la estructura presente signos de falla, además las metodologías tienen en cuenta la variabilidad de las propiedades de los elementos estructurales lo cual trae consigo una modelación mucho más ajustada a lo que ocurre en realidad en las secciones seleccionadas para el presente estudio.

\section{Referencias}

[1] CCP-14, "Norma Colombiana de Diseño de Puentes CCP-14," Asociación Colombiana de Ingeniería Sísmica, 2014.

[2] CCDSP-95, "Código Colombiano de Diseño Sísmico de Puentes CCDSP-95," Asociación Colombiana de Ingeniería Sísmica, 1995.

[3] INVIAS, "Manual de Drenaje para Carreteras," Instituto Nacional de Vías, Bogotá, 2009.

[4] M. Salgado-Gálvez, G. Bernal, and O.D. Cardona, "Evaluación Probabilista de la Amenaza Sísmica de Colombia con fines de Actualización de la Norma Colombiana de Diseño de Puentes CCP-14," Revista Internacional de Métodos Numéricos para Cálculo y Diseño en Ingeniería, vol.32, no.4, pp. 230-239, 2016.

[5] L. Perez-Rocha and M. Ordaz-Schroeder, "Maxima Earthquakes for Seismic Design of Structures," the 14th world conference on earthquake engineering, 2008.

[6] M. Ozcelik and O. Tutus, "An Investigation on Botan Bridge (Siirt - Turkey) Collapse During Construction," Structures, vol.25, pp. 268-273, 2020.

[7] J. Wilches, H. Santa Maria, R. Ridell and C. Arrate, "Effects of Changes in Seismic Design Criteria in the Transverse and Vertical Response of Chilean Highway Bridges," Engineering Structures, vol.191, pp. 370-385, 2019.

[8] G. Ahmed and O. Aziz, "Shear Strength of Joints in Precast Posttensioned Segmental Bridges During 1959-2019, Review and Analysis," Structures, vol.20, pp. 527-542, 2019.

[9] A. Memari, H. Harris, A. Hamid and A. Scanlon, "Ductility Evaluation for Typical Existing R/C Bridge Columns in the Eastern USA," Engineering Structures, vol.27, pp. 203-212, 2005. 
[10] M. Breccolotti, L. Materazzi and B. Regnicoli, "Curvature Ductility of Biaxially Loaded Reinforced Concrete Short Columns," Engineering Structures, vol.200, 109669, 2019.

[11] L. Farguier "Rehabilitation and Lessons Learned from the Collapse of Viaduct 1 Located on the Caracas-LaGuaira Highway in Venezuela," Structural Engineering International, vol.27, no.3, pp. 380-387, 2014.

[12] Q. Wang and M. Zhai, "Fatigue Performance Evaluation of Existing Concrete Girder Bridges," Structural Engineering International, vol.28, no.3, pp. 280-287, 2018.

[13] E. Muñoz and D. Gómez, "Analysis of the Evolution of Damage in the Bridges of Colombia," Revista Ingeniería de Construcción RIC, vol.28, no.1, pp. 37-62, 2013.

[14] S. Dhakal and M. Moustafa, "Moment-Curvature Analysis for Beams with Advanced Materials," SoftwareX, vol.9, pp. 175-182, 2019.

[15] S. Dolati and M. Maleki, "Ductile Behavior of Existing Internal end Diaphragms in Steel Tub Girder Bridges," Journal of Constructional Steel Research, vol.153, pp. 356-371, 2019.

[16] A. Viviescas, J. Carrillo, and L. Vargas, "Línea Base para el Monitoreo de Salud Estructural del Puente Gómez Ortiz a partir de Pruebas de Vibración Ambiental," INGE CUC, vol.14, no.1, pp. 52-65, 2018.

[17] L. Perez-Rocha and M. Ordaz-Schroeder, "Ductility Evaluation from Laboratory and Analytical Testing," the 9th world conference on earthquake engineering, 1988.

[18] SeismoSoft, "SeismoStruct," Manual de Usuario SeismoStruct (version 2016), Italy: SeismoSoft, Pavia., 2016.

[19] J. Mander, M. Priestley, and R. Park, "Theoretical Stress-Strain Model for Confined Concrete," ASCE Journal of Structural Engineering, vol.114, no.8, pp. 1804-1826, 1988.

[20] M. Priestley, "Displacement Based Seismic Design of Structures," IUSS PRESS: Pavia, 2007.

[21] M.B. Calvacanti and B. Horowitz, "Flexural Analysis of Prestressed Concrete Structures," Ibracon Structures and Materials Journal, vol.1, pp. 331-364, 2008.

[22] J.S. Du, F. Au, Y. Cheung and A. Kwan, "Ductility Analysis of Prestressed Concrete Beams with Unbonded Tendons," Engineering Structures, vol.30, no.1, pp. 13-21, 2007.

[23] R.O. Hamburger, "Defining Performance Objectives," International Workshop on Seismic Design, Methodologies for the Next Generation of Codes, 1997.

[24] D. Sosa, D. Arévalo, E. David Mora, M. Belén Correa, D. Albuja and C. Gómez, "Experimental and Analytical Study of Slender Reinforced Concrete Shear Wall under Cyclic In-Plane Lateral Load," Mathematical Problems in Engineering, vol.17, pp. 1-14, 2017.

[25] C. Cismasiu, F. Amarante, R. Da Silva, Vasco M. S. Bernardo and Paulo X. Candeias and Alexandra R. Carvalho and L. Guerreiro, "Seismic Vulnerability Assessment of a RC Pedestrian Crossing," Journal of Earthquake Engineering, vol.24, no.5, pp. 727-744, 2020.

[26] R. Sousa, J. Almeida, A. Correia and R. Pinho, "Shake Table Blind Prediction Tests: Contributions for Improved Fiber-based Frame Modelling," Journal of Earthquake Engineering, pp. 1-42, 2018.

[27] N. Serdar and R. Folic, "Vulnerability and Optimal Probabilistic Seismic Demand Model for Curved and Skewed RC Bridges," Engineering Structures, vol.176, pp. 411-425, 2018.

[28] D. Kar and R. Roy, "Seismic Behavior of RC Bridge Piers under Bidirectional Excitations: Implications of Site Effects," Journal of Earthquake Engineering, vol.22, no.2, pp. 303-331, 2018.

[29] D. Raihan and A. Rahman, "Lateral Strength and Safety Evaluation of Piers of Kadamtali Flyover in Chittagong, Bangladesh," International Journal of Advanced Structures and Geotechnical Engineering, vol.6, no.2, pp. 1-12, 2017.

[30] S. Aaleti, S. Sritharan and A. Abu-Hawash, "Innovative UHPC-normal Concrete Composite Bridge Deck" RILEM-fib-AFGC Int. Symposium on Ultra-High Performance Fibre-Reinforced Concrete, 2013.

[31] K. Thompson and R. Park, "Ductility of Prestressed and Partially Prestressed Concrete beam Sections," PCI Journal-New Zealand, 1980.

[32] AASHTO, "AASHTO LRFD Bridge Design Specifications," American Association of State Highway Transportation Officials, Washington, DC, 2014.
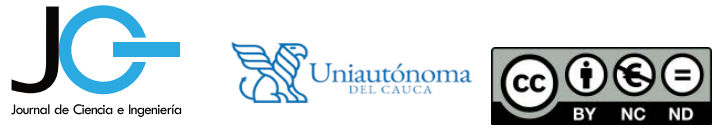